\title{
A hybrid two-step-model based on Cuckoo-Search and Grey Wolf Optimiser ensemble-based classifier approach for Network Intrusion Detection
}

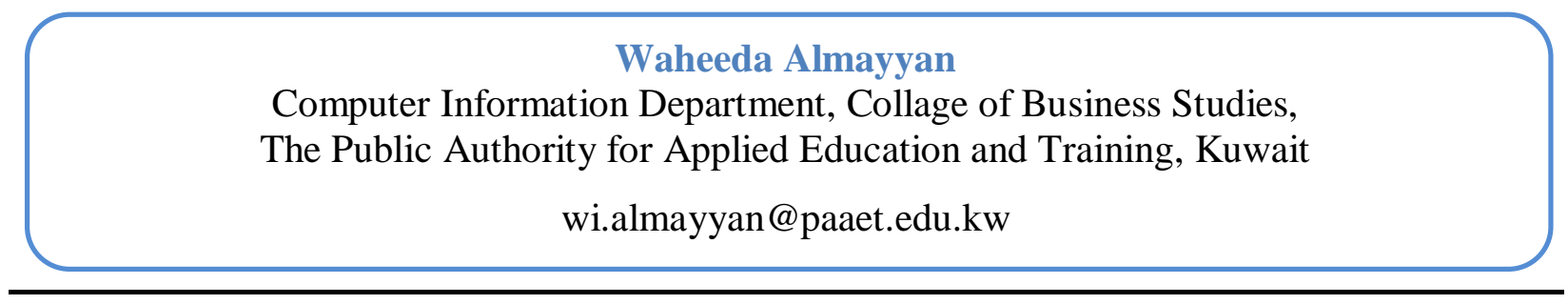

\section{ABSTRACT}

The new generation of Internet threats brought higher risks over network connectivity. This research suggests a layered feature selection Intrusion Detection System with an ensemble learning algorithms to enhance the decision about possible intrusion attacks. Cuckoo Search and Grey Wolf Optimiser approaches have been proposed to inherit their advantages to select the most distinctive features. The proposed classification model is combined with five classifiers, Fuzzy Unordered Rule Induction Algorithm, Voted Perceptron, Simple Logistic, Forest by Penalizing Attributes and C4.5 machine learning algorithms, to form a single voting-based ensemble classifier. The result indicated that the performance of the proposed ensemble based on the maximum of probabilities rule is better than the individual classifiers.

Key words:Machine Learning, Intrusion Detection Systems, Classification, Feature Selection, NSL-KDD.

\section{INTRODUCTION}

The massive evolution in information technology, Internet, computer networks and the widespread of Internet applications have brought much convenience to people's daily life and workplaces. Nonetheless, it borne a lot of security issues, such as vulnerabilities and virus attacks. Computer system security is defined as "the process of protecting confidentiality, integrity and availability" [1]. Intrusion Detection System (IDS) primary mission is to identify non-authorized accesses or intrusions, and suspicious activities or threats in information system or network [2]. It is responsible for monitoring all the activities in a system or network, to alert the system or network administrator whether a suspicious intrusion has occurred or not [3]. IDS can be classified into two broad categories according to the location in the network or by detection method, as illustrated in Figure 1. Based on the source of data IDS can be classified into Host-based and Network-based.

Host-Based: This IDS type acts on the client side by reviewing and detecting the important files and screening the usage of hardware resources [5]. Accordingly, it notifies the system administrator in case of any variation in the system or client files. 
DOI: https://dx.doi.org/10.26808/rs.ca.i10v6.02 International Journal of Computer Application (2250-1797)

Issue 10 Volume 6, November- December 2020

Network-Based: This type acts on the network side to monitor and inspect the unusual and abnormal traffic attack threats like the Denial of Services [6]. Yet, Network-Based IDS has very limited visibility inside the host machines.

Another categorization is based on the detection methodology, divide the IDS to signature-based IDS and anomaly-based IDS.

Signature-Based: It works by applying pattern matching to detect unknown attack patterns. So, it compares the incoming activity with the known attacks. In case if it recognizes any matching, it blocks the connection and stop it. Signature detection also refers misuse-based detection, pattern matching, rule-based detection [7].

Anomaly-Based: It recognizes the client or networking activities abnormal behaviour by applying several statistical, data-mining, learning based techniques [8]. This approach starts with creating a profile for the regular activities. To maintain the system security, it usually compares the new system activities with the collected profile. In case if an event did not match with the normal profile, this malicious behaviour will be considered as suspicious and it will raise an alarm.

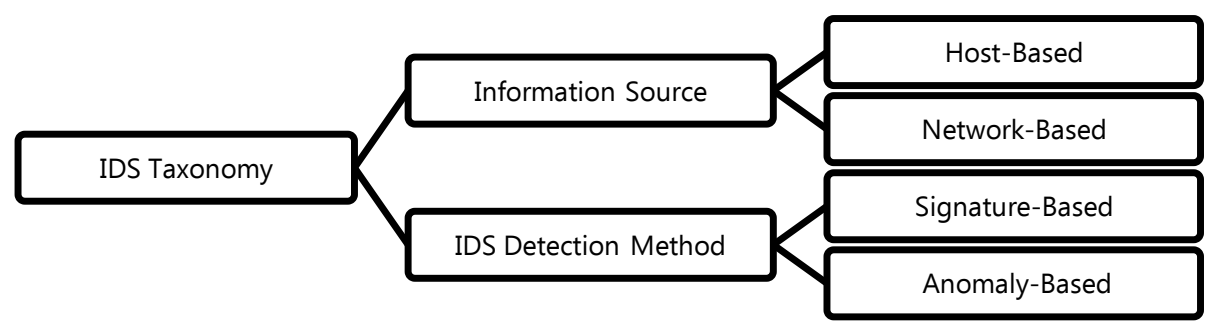

Figure 1. Categories of IDS [4]

Due to high dimensionality of audit data produced during daily routine, existing IDS are unable to detect intrusion in real time, as it results in large computational. In an experiment conducted by MIT Lincoln Lab, to statistically analyse network traffic four giga bytes of compressed binary tcp-dump data, over 7 weeks were processed into five million connection records [9].

This indicates that the high dimensionality of the data under monitoring makes the analysis of IDS very challenging. One feasible approach to reduce the computation burden is to consider feature selection algorithms. Feature selection has a major role in detecting the most significant attributes and consequently improving classification results. This research is investigates applying a hybrid two-stage algorithm based on Grey Wolf Optimizer (GWO) and Cuckoo search algorithm to find the most optimal subset and removes low rank features.

This paper is organized as follows. After the introduction, Section 2 provides a review of the literature on using data mining algorithms with IDS. Section 3 describe the research methodology. Section 4 presents experimental results and discussion followed by the conclusion in Section 5. 
DOI: https://dx.doi.org/10.26808/rs.ca.i10v6.02

International Journal of Computer Application (2250-1797)

Issue 10 Volume 6, November- December 2020

\section{RELATED WORK}

Dorothy Denning implemented the first IDS in 1986 through applying statistical models on security incidents to identify any irregular or suspicious activities [10]. Since then, many researchers applied several machine learning algorithms to tackle it.

Alomari and Othman [11] proposed a wrapper-based feature selection approach using Artificial Bee Colony algorithm (ABC) as a search strategy and using Support Vector Machine (SVM) classifier. They conducted experiments using four random subsets of KDD CUP'99 dataset, wherein each subset approximately 4000 records. The evaluation criteria were calculated based on the balance between the average accuracy and the average accuracy of selected features. Experimental results showed that the $\mathrm{ABC}+\mathrm{SVM}$ based wrapper approach achieved an overall accuracy of $99 \%$ with 8 features. Xingzhu in [12] proposed an Ant Colony Optimisation (ACO) and SVM feature weighting technique to address intrusion detection problem. Firstly, they built a complete fitness weighting index through utilising SVM grouping precision and highlighting subset measurements. Afterwards, they used the global and numerous exploration competences of ACO to construct the best solution feature list. The reported results showed that the suggested model succeeded in reducing the dimension of features with an accuracy rate of $95.75 \%$.

Rani and Xavier proposed a signature-based IDS for zero-day attacks based on combining the C5.0 classifier and One Class SVM [13]. The original dataset was used to detect the outliers using a decision function during the training session. The experiments conducted using NSLKDD Dataset showed that the approach reduced processing time, features and scored a detection rate of $99 \%$.

Ghanem and Jantan developed an ABC and particle swarm optimisation (PSO) IDS with a feedforward neural network [14]. The approach starts with generating the Pare-to optimal solutions among a set of non-dominated solutions. Next, it uses the ABC+PSO to obtain the most significant features. Acharya and Singh introduced an unsupervised intelligent water drop (IWD) algorithm and SVM to classify KDD CUP'99 dataset [15]. Results showed that the proposed model (IWD+SVM) substantially helped in reducing the features from 41 to 9 with an accuracy rate of $99.09 \%$. In the study of [16] a random forest model is proposed to detect various networking attacks on the NSL-KDD Dataset. In addition, the random forest classifier was associated with Gini feature importance for an explicit feature elimination. The experiential results showed that the proposed model achieved an accuracy of $99.88 \%$ with 25 features.

In the work of [17], a layered architecture selects the classifier based on attack type using NSLKDD dataset. After data pre-processing, Cfs Subset Eval and Wrapper Subset Eval were applied with different classification algorithms to achieve high accuracy results in recognition all attack types. Another IDS model that is based on a hybrid feature selection and clustering is developed by [18]. The approach was based oncombining K-Medoids clustering algorithm and SVM to select features. The proposed model is evaluated with KDD CUP' 99 dataset using Naïve Bayes classifier. The results of the experiments indicated that the proposed approach scored an accuracy of $91.5 \%$ and detection rate of $90.1 \%$.

Zhou and Cheng proposed an ensemble correlation-based Bat algorithm to solve intrusion detection problem [19]. Meanwhile, the ensemble classifier is built using C4.5, Random Forest and Forest by Penalizing Attributes (Forest PA) based on the average of probabilities rule. Experiments showed that the suggested approach recorded an accuracy result of $96.76 \%$ and a detection rate of $94.04 \%$. A feature selection and clustering model for IDS based on decision tree classifier is implemented by combing filter and wrapper mechanism was developed by [20]. The wrapper method is based on linear correlation coefficient algorithm and the filter method applies 
DOI: https://dx.doi.org/10.26808/rs.ca.i10v6.02 International Journal of Computer Application (2250-1797)

Issue 10 Volume 6, November- December 2020

the cuttlefish algorithm. Results obtainedover the KDD Cup'99 dataset indicated that the suggested model achieved an accuracy of $95.03 \%$ and a detection rate of $95.23 \%$.

As most of the techniques in the literature try to tackle the IDS through applying data mining techniques, in this paper we suggest a combination of feature selection method (GWO+Cuckoo). The first phase tries to eliminate irrelevant features from the original dataset. While the second phase decreases the search range from the entire original feature space to the pre-selected features. This work aims to achieve higher accuracy rates compared to the literature works.

\section{MATERIALS AND METHODS}

\section{a. NSL-KDD Dataset}

NSL-KDD dataset is an enhanced version of the original KDD CUP'99 dataset which contains proportionate to $10 \%$ of the original dataset. It was proposed by [21] with removing redundant records and saving reasonable numbers of instances so that classifier doesn't show biased results. NSL-KDD dataset comprises of four components, KDDTrain+,KDDTest+, 20\%KDDTraining+ and KDDTest-21[22].

For experimental purpose, we have used KDDTraining20\%and KDDTest+ benchmark datasets to evaluate the proposed model which consists of 25,192 and 22,544 records respectively. Each record in dataset consists of 41 features labelled as normal or attack. Features are divided into four categories [22]: basic, content, time-based, and host-based features, as shown in Table 1. Even though the NSL-KDD dataset had a few issues, it is an extremely successful dataset that can be utilized for research purposes [23].

Table 1 List of features in the NSL-KDD data set [23]

\begin{tabular}{|l|l|l|l|l|l|l|l|}
\hline \multicolumn{2}{|c|}{ Basic Features } & \multicolumn{2}{|c|}{ Content Features } & \multicolumn{2}{c}{$\begin{array}{c}\text { ime-Based Network } \\
\text { Traffic Statistics }\end{array}$} & \multicolumn{2}{c}{$\begin{array}{c}\text { Host-based Network Traffic } \\
\text { Statics }\end{array}$} \\
\hline 1. & Duration & 10. & Hot & 23. & count & 32. & dst_host_serror_rate \\
\hline 2. & Protocol_type & 11. & num_failed_logins & 24. & srv_count & 33. & dst_host_rerror_rate \\
\hline 3. & Service & 12. & logged_in & 25. & srv_serror_rate & 34. & dst_host_same_src_port_rate \\
\hline 4. & Flag & 13. & num_compromised & 26. & srv_rerror_rate & 35. & dst_host_srv_serror_rate \\
\hline 5. & Src_bytes & 14. & root_shell & 27. & rerror_rate & 36. & dst_host_count \\
\hline 6. & Dst_bytes & 15. & su_attempted & 28. & serror_rate & 37. & dst_host_srv_count \\
\hline 7. & land & 16. & num_root & 29. & same_srv_rate & 38. & dst_host_diff_srv_rate \\
\hline 8. & Wrong_fragment & 17. & num_file_creations & 30. & diff_srv_rate & 39. & dst_host_same_srv_rate \\
\hline 9. & urgent & 18. & num_shells & 31. & srv_diff_host_rate & 40. & dst_host_srv_rerror_rate \\
\hline & & 19. & num_access_files & & & 41. & dst_host_srv_diff_host_rate \\
\hline & & 20. & num_outbound_cmds & & & & \\
\hline & & 21. & is_host_login & & & & \\
\hline & & 22. & is_guest_login & & & & \\
& & & & & & \\
\hline
\end{tabular}

\section{b. Methodology}

The proposed approach is developed in four phases: data processing, feature selection, model selection, and model validation. The first phase includes data cleaning, standardization and normalization. Second phase suggests applying a hybrid algorithm based on GWO and Cuckoo search algorithm to find an optimal subset and removes low rank features. In the third stage, the data are divided into validation, training and test set. In the last phase, the performance of the selected model using the original and improved feature subset in combination with different ML classifiers is validated based on the maximum of probabilities rule. The overall framework is illustrated in Figure 2. 
DOI: https://dx.doi.org/10.26808/rs.ca.i10v6.02

International Journal of Computer Application (2250-1797)

Issue 10 Volume 6, November- December 2020

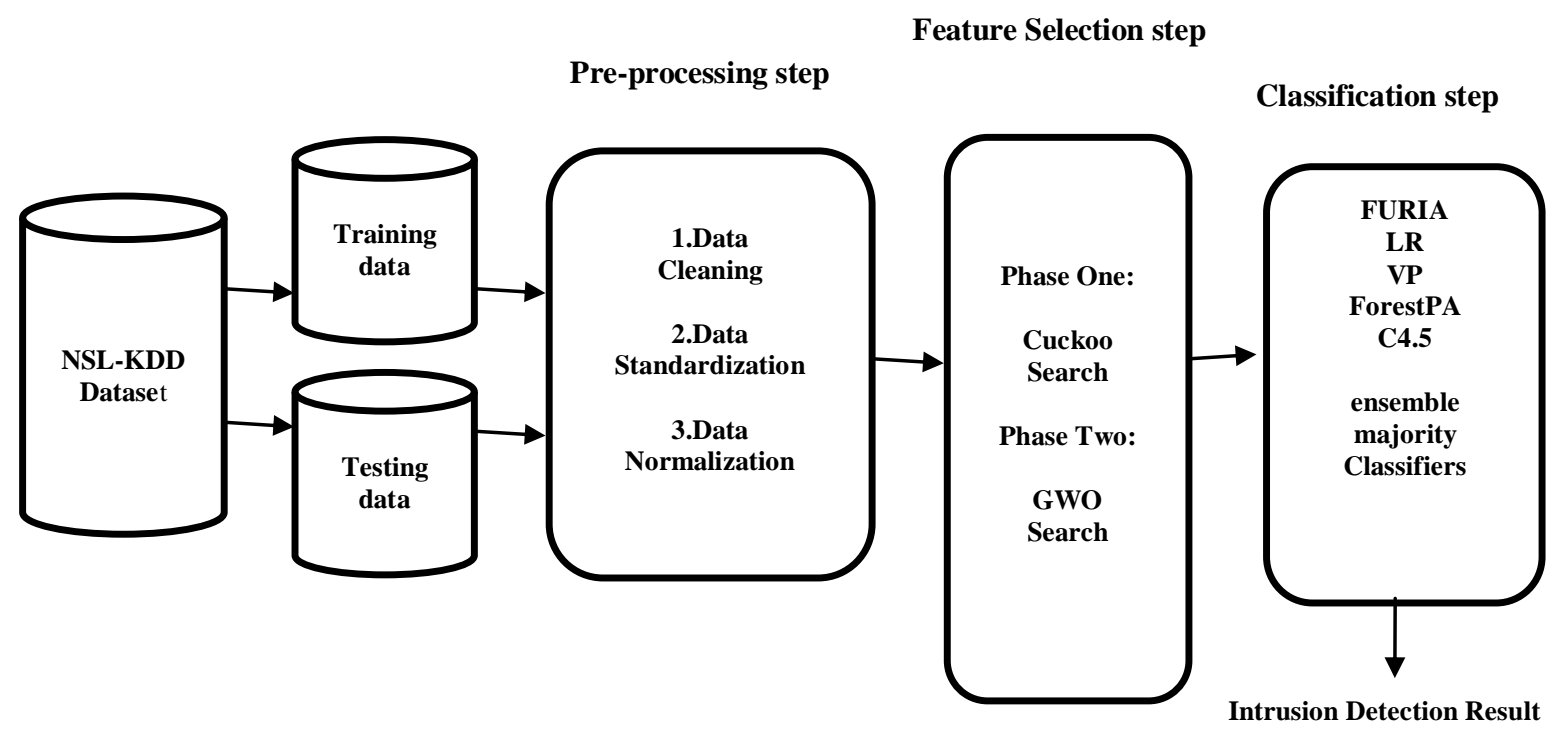

Figure 2. The Proposed Model for Feature Selection and Classification

a. Cuckoo Search via Lévy flights

Cuckoo search is a metaheuristic algorithm that has been extensively used in computational models and in adaptive search strategies for solving optimization problems [24]. One of the most desirable features of the Cuckoo search algorithm it is computationally efficient and easy to implement with a smaller number of parameters [25].

The basic idea behind the Cuckoo search algorithm is derived from the breeding behaviour of some cuckoo species. These species choose to put eggs in a randomly chosen nests of other host birds, which have similar colour and pattern of the hosts' own eggs to reduce the probability of the hosts discovering them. The cuckoos rely on these host birds to accommodate their eggs. Sometimes, some of the host birds discover the foreign eggs and throw it away or simply leave their nests.

The Cuckoo search algorithm generates a new candidate solution (nest) $\mathrm{x}_{\mathrm{i}}{ }^{(\mathrm{r}+1)}$ for a cuckoo $n$ $[26,27]:$

$\mathrm{x}_{\mathrm{i}}^{(\mathrm{r}+1)}=\mathrm{x}_{\mathrm{i}}^{(\mathrm{r})}+\alpha \otimes \operatorname{Lévy}(\lambda)$

where $\mathrm{s}$ is the step size, and $\alpha>0$ is the step size scaling. In most cases, $\alpha$ is set to 1 . The symbol $\bigoplus$ is an entry-wise multiplication that is similar to those used in PSO algorithm. Basically, Lévy flights provide a random walk, while their random steps are drawn from a Lévy distribution for large steps:

$\operatorname{Lévy}(\lambda) \sim \mathrm{u}=\mathrm{s}^{-\lambda},(1<\lambda \leq 3)$

This makes the Cuckoo search more efficient in exploring the search space as it guaranteed that the algorithm will not fall into a local optimum trap [24].

\section{b. Grey Wolf Optimization Algorithm}

The GWO algorithm is a metaheuristic algorithm that was introduced by Mirjalili in 2014 [28]. The GWO algorithm is primarily motivated by the leadership and hunting behaviors of grey 
wolves in nature, which mostly tend to live in packs consisting of 5-12 with strict hierarchy, and each wolf in the group has a defined role in the hunting process [29]. Each herd has four main ranks that are modeled as a pyramid [30]. The first level in the hierarchy of grey wolves is the leader of pack and is called alpha $(\alpha)$. This level is responsible for decisions about all wolf behaviors. Beta $(\beta)$ wolves, is the second level and are responsible in helping in some choices in the packs. The third level is the delta $(\delta)$ wolves and are responsible for watching the boundaries of territory, protecting the group, hunting, and so on. The last level in the hierarchal model is called omega $(\omega)$, which is the weakest of the wolves, as it should obey other individuals' orders [29].

The second inspiration of GWO is the fact that wolves are known for their group-hunting strategy to catch prey. The hunting process starts with tracking and chasing the prey, then encircling and harassing until it finally stops moving before attacking in a manner that can be mathematically modeled as:

$\vec{D}=\left|\vec{C} \cdot \overrightarrow{X_{p}}-\vec{X}(t)\right|$,

$\vec{X}(t+1)=\left|\overrightarrow{X_{p}}(t)-\vec{A} \cdot \vec{D}\right|$

where $\vec{X}$ and $\overrightarrow{X_{p}}$ represent the positions of the prey and grey wolf at an iteration (t), respectively. $\vec{A}$ and $\vec{C}$ are coefficient vectors that can be formulated as follows:

$\vec{A}=\left|2 a \cdot \overrightarrow{\text { rand }_{1}}-\vec{a}\right|$,

$\vec{C}=2 . \overrightarrow{\text { rand }_{2}}$

where $\overrightarrow{\operatorname{rand}_{1}}$ and $\overrightarrow{\text { rand }_{2}}$ are random vectors in $[0,1] . \vec{a}$ is linearly decreasing from 2 to zero over iterations as follows:

$a=2-t * \frac{2}{\text { iterations }}$

The position of the grey wolf $(X, Y)$ can be updated according to the position of the prey $\left(X^{*}, Y^{*}\right)$. The vectors $\vec{A}$ and $\vec{C}$ used to update the position of the best grey wolf. As the alpha, beta, and delta indicate the best three solutions, the rest of the wolves update their positions according to the best three solutions $\left(\overrightarrow{X_{1}}, \overrightarrow{X_{2}}\right.$, and $\left.\overrightarrow{X_{3}}\right)$. This can be expressed as follows:

$\vec{X}(t+1)=\left(\overrightarrow{X_{1}}+\overrightarrow{X_{2}}+\overrightarrow{X_{3}}\right) / 3$,

$\overrightarrow{X_{1}}=\overrightarrow{X_{\propto}}-\overrightarrow{A_{1}} \cdot\left(\overrightarrow{D_{\propto}}\right) \cdot \overrightarrow{D_{\propto}}=\left|\vec{C} \cdot \overrightarrow{X_{\propto}}-\vec{X}\right| \quad$,

$\overrightarrow{X_{2}}=\overrightarrow{X_{\beta}}-\overrightarrow{A_{2}} \cdot\left(\overrightarrow{D_{\beta}}\right) \cdot \overrightarrow{D_{\beta}}=\left|\overrightarrow{C_{2}} \cdot \overrightarrow{X_{\beta}}-\vec{X}\right|$,

$\overrightarrow{X_{3}}=\overrightarrow{X_{\delta}}-\overrightarrow{A_{3}} \cdot\left(\overrightarrow{D_{\delta}}\right) \cdot \overrightarrow{D_{\delta}}=\left|\overrightarrow{C_{3}} \cdot \overrightarrow{X_{\delta}}-\vec{X}\right|$

\section{c. On Ensemble Classifiers}

Ensemble learning is a paradigm applied in numerous classification problems where several individual classification base models are combined to obtain one decision. Ensemble methods' main objective is to achieve more accurate classification rates than a single model usually accomplishes [31]. Ensemble methods try to train multiple learning machines and combine their predictions to obtain better accuracy. This paradigm is powerful in achieving an estimation result with higher stability and accuracy by combining the decisions of multiple independent classification models [32]. As noticed in literature, several algorithms were chosen to build ensemble learning models, namely bagging, boosting, voting, Bayesian parameter averaging, and stacking [31,33].

For building classification learning model, we choose: Fuzzy Unordered Rule Induction Algorithm (FURIA), Voted Perceptron (VP), Simple Logistic (SL) and C4.5 machine learning 
algorithms. Numerous studies have proven that decision trees are very effective methods of supervised learning [33]. C4.5 has been widely used in data mining due to its high efficiency in handling continuous, discrete attributes and missing values [34]. Meanwhile, RandomForest is generally simpler and can achieve accurate and robust results than single decision trees [35]. Moreover, ForestPA is a class implementing decision forest algorithm that generate trees with high precision based on the strength of the feature space. With its novel weight assignment strategy, bootstrap sampling, and penalized attributes, ForestPA generates highly diverse trees while retaining their higher individual accuracy [36]. FURIA classifier considered an extension of the RIPPER rule learning algorithm having its simplicityin generating unordered rule sets [37]. Moreover, it makes use of an efficient rule stretching method to deal with uncovered examples. VP is better in considering noisy or inseparable data as it approaches to small sample analysis and taking advantage of the boundary data of largest margin [38]. SL is a well-known discriminative technique for classification, which have been used in many applications $[39,40]$.

\section{RESULTS AND DISCUSSION}

Many scholars use accuracy and false positive rate, but others $[41,42,43]$ claim that accuracy and false positive rates are not enough and merely using accuracy results can be misleading. We consider using Sensitivity, Specificity, Balanced Accuracy, Precision and $f$-measure as performance measurement metrics.

\section{Data Pre-processing}

Data pre-processing is a fundamental task in data mining and usually take place before constructing the training model to ensure the quality of the raw data. Data pre-processing procedure here involves raw data cleaning, standardization, and normalization.

\section{a. Data Cleaning}

Although NSL-KDD dataset has been improved, we noticed that the value of the feature "num_outbound_cmds" is always 0 , so we decided to remove it.

\section{b. Data Standardization}

In data standardization we convert the symbolic features to numerical. The remaining 40 features, include 7 symbolic features and 33 numerical features. We started with "land", "logged_in", "is_hot_login", and "is_guest_login" features which only contain "yes" and "no", we encoded as 1 or 0 . While the categoricalfeatures, "protocol_type", "service", and "flag", which contain 3, 70, and 11 characters, respectively, one-hot encoding technique is applied.

\section{c. Data Normalization}

The main objective of data normalization is to decrease the numerical variations of different features, by scaling conditional feature value to a reasonable range. Here, we applied the MinMax normalization map the initial features values $\mathrm{v}$ from the original $\left[\min _{A}, \max _{A}\right]$ to $[0,1]$, and the normalized value $v$ ' is

$v=\left(v-\min _{A}\right) /\left(\max _{A}-\min _{A}\right)$

\section{Discussion}

Our procedure starts with first training the model using the KDDTrain+_20 dataset, once it is built the next step is to use the KDDTest+ dataset for testing the suggested model. Table 2 shows the first set of experimental results of all classifier with full set of features. It shows that the ensemble classifier model achieved superior result in terms of Specificity, classification accuracy and Precision rates as it achieved $82.7 \%, 91.4 \%$ and $78.3 \%$ respectively. Followed by the 
DOI: https://dx.doi.org/10.26808/rs.ca.i10v6.02 International Journal of Computer Application (2250-1797)

Issue 10 Volume 6, November- December 2020

ForestPA, which showed the second highest rates of accuracy, then the other classification algorithms.

Table 2. Full dataset-Comparative study of unprocessed dataset (with all the original features)

\begin{tabular}{|l|l|l|l|l|l|}
\hline Model & Sensitivity & Specificity & $\begin{array}{l}\text { Balanced } \\
\text { Accuracy }\end{array}$ & Precision & f-measure \\
\hline FURIA & 0.706 & 0.713 & 0.857 & 0.765 & 0.734 \\
\hline VP & 0.788 & 0.683 & 0.842 & 0.767 & 0.777 \\
\hline SL & 0.784 & 0.688 & 0.844 & 0.769 & 0.776 \\
\hline ForestPA & 0.540 & 0.771 & 0.885 & 0.757 & 0.630 \\
\hline C4.5 & 0.593 & 0.752 & 0.876 & 0.759 & 0.666 \\
\hline Ensemble & 0.471 & 0.827 & 0.914 & 0.783 & 0.588 \\
\hline
\end{tabular}

For the first feature selection phase, we applied the standard Cuckoo, PSO and Elephant search algorithms and select 13,16 and 15 features respectively, among 41 features of NSL-KDD dataset. Table 3 lists the selected features with a number of selected features obtained by standard Cuckoo, PSO and Elephant search algorithms. From the table, it could be noted that the proposed Cuckoo algorithm performed the lowest number of features during the experiment runs followed by Elephant and PSO algorithms.

Table 3. Selected features in first step

\begin{tabular}{|c|c|c|}
\hline Method & No. Of Features & Selected Features \\
\hline Cuckoo & 13 & $\begin{array}{l}\text { src_bytes } \\
\text { dst_bytes } \\
\text { wrong_fragment } \\
\text { logged_in } \\
\text { num_compromised } \\
\text { serror_rate } \\
\text { srv_serror_rate } \\
\text { same_srv_rate } \\
\text { diff_srv_rate } \\
\text { srv_diff_host_rate } \\
\text { dst_host_srv_diff_host_rate } \\
\text { dst_host_srv_serror_rate } \\
\text { flag }\end{array}$ \\
\hline PSO & 16 & $\begin{array}{l}\text { src_bytes } \\
\text { dst_bytes } \\
\text { land } \\
\text { wrong_fragment } \\
\text { logged_in } \\
\text { num_root } \\
\text { serror_rate } \\
\text { srv_serror_rate } \\
\text { same_srv_rate } \\
\text { diff_srv_rate } \\
\text { srv_diff_host_rate } \\
\text { dst host same srv rate }\end{array}$ \\
\hline
\end{tabular}


DOI: https://dx.doi.org/10.26808/rs.ca.i10v6.02 International Journal of Computer Application (2250-1797)

Issue 10 Volume 6, November- December 2020

\begin{tabular}{|l|l|l|}
\hline & & dst_host_srv_diff_host_rate \\
& & dst_host_srv_serror_rate \\
service & flag \\
\hline & 15 & src_bytes \\
& dst_bytes \\
& wrong_fragment \\
& logged_in \\
& num_shells \\
& count \\
& srv_serror_rate \\
& same_srv_rate \\
& diff_srv_rate \\
& dst_host_srv_count \\
& dst_host_srv_diff_host_rate \\
& dst_host_serror_rate \\
& dst_host_srv_serror_rate \\
& service \\
& flag \\
&
\end{tabular}

After constructing feature sets using various settings, either Cuckoo, PSO or Elephant these features were fed into individual and ensemble classifiers. Tables 4-6 present the result of classification performance of FURIA, VP, SL, ForestPA, C4.5 and the ensemble classifier over the obtained dataset from Cuckoo, PSO and Elephant search algorithms respectively. The highest Specificity, Balanced Accuracy and Precision readings were scored with the Cuckoo search algorithm with 13 features.

We also noticed that the ensemble classifier in all cases obtained the best results over individual classifiers in terms of Specificity, Balanced Accuracy and Precision. Up to this point, feature selection using Cuckoo search algorithm gives better result than other heuristic search algorithms. According to the Venn diagram analysis (Figure 2) showing that there are 10 common features between Cuckoo, PSO and Elephant metasearch. It includes: src_bytes, dst_bytes, wrong_fragment ,logged_in, srv_serror_rate, same_srv_rate, diff_srv_rate, dst_host_srv_diff_host_rate, dst_host_srv_serror_rate and flag.

Table 4. Performance classification based on Cuckoo search in first step

\begin{tabular}{|l|l|l|l|l|l|}
\hline Model & Sensitivity & Specificity & $\begin{array}{l}\text { Balanced } \\
\text { Accuracy }\end{array}$ & Precision & f-measure \\
\hline FURIA & 0.424 & 0.837 & 0.919 & 0.775 & 0.548 \\
\hline VP & 0.490 & 0.818 & 0.909 & 0.780 & 0.602 \\
\hline SL & 0.491 & 0.815 & 0.908 & 0.778 & 0.602 \\
\hline ForestPA & 0.507 & 0.791 & 0.896 & 0.763 & 0.609 \\
\hline C4.5 & 0.478 & 0.825 & 0.912 & 0.783 & 0.594 \\
\hline Ensemble & 0.407 & 0.849 & 0.925 & 0.781 & 0.535 \\
\hline
\end{tabular}


DOI: https://dx.doi.org/10.26808/rs.ca.i10v6.02 International Journal of Computer Application (2250-1797)

Issue 10 Volume 6, November- December 2020

Table 5. Performance classification based on PSO search in first step

\begin{tabular}{|l|l|l|l|l|l|}
\hline Model & Sensitivity & Specificity & $\begin{array}{l}\text { Balanced } \\
\text { Accuracy }\end{array}$ & Precision & f-measure \\
\hline FURIA & 0.576 & 0.787 & 0.893 & 0.781 & 0.663 \\
\hline VP & 0.505 & 0.813 & 0.907 & 0.782 & 0.614 \\
\hline SL & 0.509 & 0.810 & 0.905 & 0.780 & 0.616 \\
\hline ForestPA & 0.588 & 0.782 & 0.891 & 0.781 & 0.671 \\
\hline C4.5 & 0.584 & 0.782 & 0.891 & 0.779 & 0.668 \\
\hline Ensemble & 0.501 & 0.819 & 0.910 & 0.786 & 0.612 \\
\hline
\end{tabular}

Table 6. Performance classification based on Elephant search in first step

\begin{tabular}{|l|l|l|l|l|l|}
\hline Model & Sensitivity & Specificity & $\begin{array}{l}\text { Balanced } \\
\text { Accuracy }\end{array}$ & Precision & f-measure \\
\hline FURIA & 0.510 & 0.812 & 0.906 & 0.782 & 0.617 \\
\hline VP & 0.494 & 0.786 & 0.893 & 0.753 & 0.597 \\
\hline SL & 0.495 & 0.786 & 0.893 & 0.753 & 0.598 \\
\hline ForestPA & 0.539 & 0.801 & 0.900 & 0.782 & 0.638 \\
\hline C4.5 & 0.528 & 0.807 & 0.904 & 0.784 & 0.631 \\
\hline Ensemble & 0.462 & 0.835 & 0.917 & 0.787 & 0.582 \\
\hline
\end{tabular}

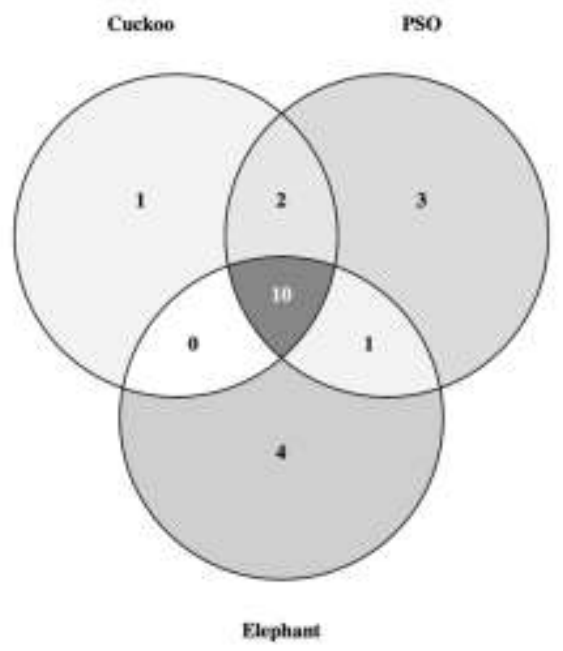

Figure 2. Venn diagram analysis of shared features between Cuckoo, PSO and Elephant search technique

In the second phase, we applied the GWO algorithm over the reducted set of features. Tables 7 and 8 list the obtained features and the classifier's performance. Worth noting that the obtained features were among the previously mentioned common feature. The ensemble classifier obtained the best results over individual classifiers in terms of Specificity, Balanced Accuracy and Precision where it scored $83.5 \%, 91.8 \%$ and $78.3 \%$ respectively with 8 features.

Table 9 shows the summary of result pointing to the average and worst classification rates with the number of selected features for NSL-KDD. From the Table, we noticed that the proposed 
DOI: https://dx.doi.org/10.26808/rs.ca.i10v6.02 International Journal of Computer Application (2250-1797)

Issue 10 Volume 6, November- December 2020

Cuckoo+GWO algorithm achieved the second highest result for best average accuracy compared to the other heuristic search algorithms. Besides that, the proposed algorithm was superior from the other techniques in the accuracy of worst classification and the number of chosen features. So, as the proposed algorithm worked on a smaller number of features compared to the original dataset, it is obvious that it was efficient enough to maintain a fair performance. Table 10 illustrates the comparison of our proposed approach with other approaches. Obviously, the proposed algorithm obtained significant result compared to approaches in literature in terms of classification accuracy and number of selected features.

Table 7. Selected features based on Cuckoo+GWO in second step

\begin{tabular}{|l|l|l|}
\hline Method & No. Of Features & Selected Features \\
\hline Cuckoo+GWO & $\mathbf{8}$ & dst_bytes \\
& & wrong_fragment \\
& & logged_in \\
& & srv_serror_rate \\
& same_srv_rate \\
& & diff_srv_rate \\
& dst_host_srv_diff_host_rate \\
& & flag \\
\hline
\end{tabular}

Table8. Performance classification based on Cuckoo+GWO in second step

\begin{tabular}{|l|l|l|l|l|l|}
\hline Model & Sensitivity & Specificity & $\begin{array}{l}\text { Balanced } \\
\text { Accuracy }\end{array}$ & Precision & f-measure \\
\hline FURIA & 0.524 & 0.789 & 0.895 & 0.767 & 0.623 \\
\hline VP & 0.459 & 0.828 & 0.914 & 0.779 & 0.577 \\
\hline SL & 0.464 & 0.826 & 0.913 & 0.778 & 0.581 \\
\hline ForestPA & 0.527 & 0.770 & 0.885 & 0.752 & 0.620 \\
\hline C4.5 & 0.451 & 0.835 & 0.917 & 0.783 & 0.572 \\
\hline Ensemble & 0.450 & 0.835 & 0.918 & 0.783 & 0.571 \\
\hline
\end{tabular}

Table9: Summary of average and worst classification rates

\begin{tabular}{|c|c|c|c|}
\hline Method & $\begin{array}{c}\text { Average classification } \\
\text { Accuracy }\end{array}$ & $\begin{array}{c}\text { Worst classification } \\
\text { Accuracy }\end{array}$ & $\begin{array}{c}\text { Selected } \\
\text { Features }\end{array}$ \\
\hline None & 0.870 & 0.842 & 40 \\
\hline Cuckoo & 0.912 & 0.896 & 13 \\
\hline PSO & 0.902 & 0.893 & 16 \\
\hline Elephant & 0.902 & 0.893 & 8 \\
\hline Cuckoo+GWO & 0.907 & 0.885 & \\
\hline
\end{tabular}


DOI: https://dx.doi.org/10.26808/rs.ca.i10v6.02 International Journal of Computer Application (2250-1797)

Issue 10 Volume 6, November- December 2020

Table10. Comparison of Cuckoo+GWO-ensemble and previous research results (accuracy in \%)

\begin{tabular}{|c|c|c|c|c|c|}
\hline $\begin{array}{c}\text { Author and } \\
\text { Year }\end{array}$ & $\begin{array}{c}\text { Feature } \\
\text { Selection } \\
\text { Algorithm }\end{array}$ & Dataset & $\begin{array}{c}\text { Classification } \\
\text { Algorithm }\end{array}$ & $\begin{array}{c}\text { Classification } \\
\text { Accuracy }\end{array}$ & $\begin{array}{c}\text { No. Of } \\
\text { Selected } \\
\text { Features }\end{array}$ \\
\hline $\begin{array}{c}\text { Our } \\
\text { proposed } \\
\text { approach } \\
\end{array}$ & Cuckoo+GWO & NSL-KDD & $\begin{array}{l}\text { Ensemble } \\
\text { classifier }\end{array}$ & $91.8 \%$ & 8 \\
\hline $\begin{array}{c}\text { Keshtgary, } \\
\text { Rikhtegar et } \\
\text { al., } 2018 \\
{[18]}\end{array}$ & $\begin{array}{c}\text { K- } \\
\text { Medoids+SVM }\end{array}$ & $\begin{array}{c}\text { KDD } \\
\text { CUP'99 }\end{array}$ & $\begin{array}{c}\text { Naïve Bayes } \\
\text { classifier }\end{array}$ & $91.5 \%$ & 10 \\
\hline $\begin{array}{c}\text { Zhou and } \\
\text { Cheng } \\
(2019) \\
{[19]}\end{array}$ & $\begin{array}{l}\text { Correlation- } \\
\text { based+ Bat- } \\
\text { Algorithm }\end{array}$ & NSL-KDD & $\begin{array}{c}\text { C4.5, } \\
\text { Random } \\
\text { Forest (RF) } \\
\text { and ForestPA }\end{array}$ & $96.76 \%$ & 10 \\
\hline $\begin{array}{c}\text { Mohammadi } \\
\text { et al., } 2019 \\
{[20]}\end{array}$ & $\begin{array}{c}\text { linear } \\
\text { correlation } \\
\text { coefficient }+ \\
\text { cuttlefish } \\
\text { algorithm }\end{array}$ & $\begin{array}{c}\text { KDD } \\
\text { CUP'99 }\end{array}$ & Decision tree & $95.03 \%$ & 10 \\
\hline $\begin{array}{c}\text { Negandhi et } \\
\text { al. } 2019 \\
{[16]}\end{array}$ & $\begin{array}{l}\text { Gini feature } \\
\text { importance }\end{array}$ & NSL-KDD & $\begin{array}{l}\text { random } \\
\text { forest }\end{array}$ & $99.88 \%$ & 25 \\
\hline
\end{tabular}

\section{CONCLUSION}

In this research we suggested a layered feature selection IDS with an ensemble learning algorithms to enhance the decision about possible intrusion attacks. We reduced the dimensions of the NSL-KDD dataset by using two-stage feature selection technique. Cuckoo and GWO approaches have been propose to classify network traffic, whether normal or attack. The proposed classification model was boosted by introducing ensemble learning of FURI, VP, SL, ForestPA and C4.5 machine learning algorithms. With this research topic, we managed to get good results by using ensemble learning.

\section{REFERENCES}

1. Stallings W, Brown L (2008) Computer security principals and practice. Pearson Education, Upper Saddle River.

2. Araujo J. D, Abdelouahab Z. Virtualization in Intrusion Detection System: A Study on Different Approaches for Cloud Computing Environments. International Journal of Computer Science and Network Security (IJCSNS), Vol.12, No.11, pp.9-16(2012).

3. Shen J, Wang J (2011) Network intrusion detection by artificial immune system. In: IECON proceedings (industrial electronics conference). pp 4716-4720.

4. Wolf L, Shashua A (2005) Feature selection for unsupervised and supervised inference: the emergence of sparsity in a weighted- based approach. J Mach Learn Res 6:378-384. https://doi. org/10.1109/iccv.2003.1238369

5. Vithalpura JS, Diwanji HM (2015) Analysis of fitness function in designing genetic algorithm based intrusion detection system. J Sci Res Dev 3:86-92. 
DOI: https://dx.doi.org/10.26808/rs.ca.i10v6.02 International Journal of Computer Application (2250-1797)

Issue 10 Volume 6, November- December 2020

6. Garcia-Teodoro, P., Diaz-Verdejo, J., Maciá-Fernández, G., Vázquez, E.: Anomaly-based network intrusion detection: techniques, systems and challenges. Comput. Secur. 28, 18-28 (2009).

7. Shahreza, M.L., Moazzami, D., Moshiri, B., Delavar, M.R.: Anomaly detection using a selforganizing map and particle swarm optimization. Sci. Iran. 18, 1460-1468 (2011).

8. Liao, H.-J., Lin, C.-H.R., Lin, Y.-C., Tung, K.-Y.: Intrusion detection system: a comprehensive review. J. Netw. Comput. Appl. 36, 16-24 (2013).

9. Lippmann, Richard, Joshua W. Haines, David J. Fried, Jonathan Korba, and Kumar Das. "The 1999 DARPA off-Line Intrusion Detection Evaluation." Comput. Netw. 34, no. 4 (October 2000): 579-595. doi:10.1016/S1389-1286(00)00139-0.

10. Dorothy Elizabeth Denning, "An Intrusion-Detection Model," in IEEE Transactions on Software Engineering, vol. SE-13, no. 2, pp. 222- 232, Feb. 1987.

11. Alomari O, Othman ZA (2012) Bees algorithm for feature selection in network anomaly detection $\beta$-Hill climbing for optimization problems view project feature selection on highdimensional data view project. Artic J Appl Sci Res 8:1748-1756.

12. Xingzhu W (2015) ACO and SVM selection feature weighting of net- work intrusion detection method. Int J Secur its Appl 9:259-270. https://doi.org/10.14257/ijsia.2015.9.4.24

13. Rani MS, Xavier SB (2015) A hybrid intrusion detection system based on C5. 0 decision tree and one-class SVM [J]. Int J CurrEng Technol 5:2001-2007.

14. Ghanem WAHM, Jantan A (2016) Novel multi-objective artificial bee colony optimization for wrapper based feature selection in intructiondetectoin. Int J Adv Soft Comput its Appl 8:70-81

15. Acharya N, Singh S (2018) An IWD-based feature selection method for intrusion detection system. Soft Comput 22:4407-4416. https://doi.org/10.1007/s00500-017-2635-2.

16. Negandhi P, Trivedi Y, Mangrulkar R (2019) Intrusion detection system using random forest on the NSL-KDD dataset. Emerging research in computing. Information communication and applications. Springer, Berlin, pp 519-531.

17. Çavuşoğlu Ü (2019) A new hybrid approach for intrusion detection using machine learning methods. ApplIntell 49:2735-2761. https ://doi.org/10.1007/s10489-018-01408-x.

18. Keshtgary, M., Rikhtegar, N., et al. (2018). Intrusion detection based on a novel hybrid learning approach. Journal of AI and Data Mining, 6(1), 157-162.

19. Zhou, Y.-Y., \& Cheng, G. (2019). An efficient network intrusion detection system based on feature selection and ensemble classifier. arXiv preprint arXiv:1904.01352.

20. Mohammadi, S., Mirvaziri, H., Ghazizadeh-Ahsaee, M., \&Karimipour, H. (2019). Cyber intrusion detection by combined feature selection algorithm. Journal of Information Security and Applications, 44, 80-88.

21. Dhanabal L, Shantharajah DSP (2015) A Study On NSL-KDD dataset for intrusion detection system based on classification algorithms. Int J Adv Res ComputCommunEng 4:446-452. https://doi. org/10.17148/IJARCCE.2015.4696.

22. P. Mishra, V. Varadharajan, U. Tupakula, and E. S. Pilli, "A detailed investigation and analysis of using machine learning techniques for intrusion detection," IEEE Commun. Surveys Tuts., vol. 21, no. 1, pp. 686-728, 1st Quart., 2019.

23. A. Shrivastava, J.Sondhi and S. Ahirwar, "Cyber attack detection and classification based on machine learning technique using nslkdd dataset", Int. Reserach J. Eng. Appl. Sci., vol.5, issue2, pp.28-31, 2017. 
DOI: https://dx.doi.org/10.26808/rs.ca.i10v6.02 International Journal of Computer Application (2250-1797)

Issue 10 Volume 6, November- December 2020

24. Xin-She Yang and Suash Deb. Cuckoo search via Lévy flights. In Nature \& Biologically Inspired Computing, 2009. NaBIC 2009. World Congress on, pages 210-214. IEEE, 2009.

25. Xin-She Yang and Suash Deb, "Cuckoo search: recent advances and applications," Neural Computing and Applications, vol. 24, no. 1, pp. 169-174, 2014.

26. P. Civicioglu and E. Besdok, "A conceptual comparison of the Cuckoo-search, particle swarm optimization, differential evolution and artificial bee colony algorithms," Artificial Intelligence Review, vol. 39, no. 4, pp. 315-346, 2013.

27. D. Rodrigues, L. A. Pereira, T. Almeida, J. P. Papa, A. Souza, C. C. Ramos and X.-S. Yang, "BCS: A Binary Cuckoo Search algorithm for feature selection," in 2013 IEEE International Symposium on Circuits and Systems (ISCAS2013), 2013.

28. S. Mirjalili, S.M. Mirjalili, A. Lewis, Grey wolf optimizer, Adv. Eng. Software 69 (2014) 46-61.

29. Alzubi QM, Anbar M, Alqattan ZNM et al (2019) Intrusion detection system based on a modified binary grey wolf optimisation. Neural ComputAppl1:1-13. https://doi.org/10.1007/s00521-019-04103-1.

30. Emary E, Zawbaa HM (2016) Impact of chaos functions on modern swarm optimizers. PLoS One 11:1-26.

31. Wozniak, M.; Graña, M.; Corchado, E. A survey of multiple classifier systems as hybrid systems. Inf. Fusion 2014, 16, 3-17.

32. C. Zhang and Y. Ma, Ensemble Machine Learning: Methods and Applications. New York, NY, USA: Springer, 2012.

33. Wozniak, M.; Graña, M.; Corchado, E. A survey of multiple classifier systems as hybrid systems. Inf. Fusion 2014, 16, 3-17.

34. G. Rahman and Z. Islam, A decision tree-based missing value imputation technique for data pre-processing, in Proc. 9th Australas. Data Mining Conf., vol. 121, 2011, pp. 41-50.

35. R. Polikar, Ensemble based systems in decision making, IEEE Circuits and Systems Magazine 6(3) (2006) 21.

36. Adnan, M.N., and Islam, M.Z. (2017). Forest PA: Constructing a decision forest by penalizing attributes used in previous trees. Expert Systems with Applications, 89, 389-403. doi:10.1016/j.eswa.2017.08.002

37. J. C. Huhn and E. Hullermeier. FURIA: an algorithm for unordered fuzzy rule induction. Data Mining and Knowledge Discovery, 19(3):293-319, 2009.

38. Fruend Y. and Schapire R. (1999). Large Margin Classification Using the Perceptron Algorithm. Machine Learning, 37(3), 277-296.

39. Hastie T, Tibshirani R, Friedman J. The elements of statistical learning. New York, NY: Springer-Verlag; 2001.

40. C.M.Bishop, Pattern Recognition and Machine Learning. NewYork, NY, USA: Springer, 2006.

41. Davis, Jesse, and Mark Goadrich. "The Relationship between Precision- Recall and ROC Curves." In Proceedings of the 23rd International Conference on Machine Learning, 233240. ICML '06. New York, NY, USA: ACM, 2006. doi:10.1145/1143844.1143874.

42. Kotsiantis, S. B. "Supervised Machine Learning: A Review of Classification Techniques." In Proceedings of the 2007 Conference on Emerging Artificial Intelligence Applications in Computer Engineering: Real Word AI Systems with Applications in eHealth, HCI, Information Retrieval and Pervasive Technologies, 3-24. Amsterdam, The Netherlands, The Netherlands: IOS Press, 2007. 
DOI: https://dx.doi.org/10.26808/rs.ca.i10v6.02 International Journal of Computer Application (2250-1797) Issue 10 Volume 6, November- December 2020

43. Williams, Nigel, Sebastian Z, and Grenville Armitage. "A Preliminary Performance Comparison of Five Machine Learning Algorithms for Practical IP Traffic Flow Classification." Computer Communication Review 30 (2006). 